\title{
IMPROVEMENTS IN PROCEDURAL BLANKS AT NOSAMS: REFLECTIONS OF IMPROVEMENTS IN SAMPLE PREPARATION AND ACCELERATOR OPERATION
} \author{
A. P. MCNICHOL, A. R. GAGNON, E. A. OSBORNE, D. L. HUTTON, K. F. VON REDEN
and R. J. SCHNEIDER
}

National Ocean Sciences Accelerator Mass Spectrometer Facility, Department of Marine Geology and Geophysics, Woods Hole Oceanographic Institution, Woods Hole, Massachusetts 02543 USA

\begin{abstract}
During the four years the Sample Preparation Laboratory (SPL) at the National Ocean Sciences Accelerator Mass Spectrometer (NOSAMS) Facilty has been in operation we have accumulated much data from which we can assess our progress. We evaluate our procedural blanks here and describe modifications in our procedures that have improved our analyses of older samples. In the SPL, we convert three distinct types of samples-seawater, $\mathrm{CaCO}_{3}$ and organic carbon-to $\mathrm{CO}_{2}$ prior to preparing graphite for the accelerator and have distinct procedural blanks for each procedure. Dissolved inorganic carblanks" by processing from acidified seawater samples by sparging with a nitrogen carrier gas. We routinely analyze "line is prepared by acidifying in $\mathrm{CO}_{2}$ from a ${ }^{14} \mathrm{C}$-dead source through the entire stripping procedure. Our hydrolysis blank, IAEA C-1, graphite, NBS-21, or a commercially available $\mathrm{H}_{3} \mathrm{PO}_{4}$ at $60^{\circ} \mathrm{C}$ overnight, identical to our sample preparation. We use a dead busted at $850^{\circ} \mathrm{C}$ for 5 cormer organic combustion blank; organic samples are comprocedure contributes the major portion of the oxidant. Analysis of our water stripping data suggests that one step in the procedure contributes the major portion of the line blank. At present, the contribution from the line blank has no effect on our seawater analyses (fraction modern ( $\mathrm{fm}$ ) between 0.7 and 1.2). Our hydrolysis blanks can have an fm value as low as 0.0006 , tinely achieved inely between 0.0020 and 0.0025 . The fm of our best organic combustion blanks is higher than those routinely achieved in other laboratories and we are currently altering our methods to reduce it.
\end{abstract}

\section{INTRODUCTION}

Studies of the ocean carbon cycle, water-mass circulation and paleoceanography provide the Sample Preparation Laboratory (SPL) at the National Ocean Sciences Accelerator Mass Spectrometry (NOSAMS) Facility with three general types of samples-dissolved inorganic carbon $\left(\Sigma \mathrm{CO}_{2}\right)$ in seawater, $\mathrm{CaCO}_{3}$ as foraminifera and other shells, and organic carbon in many different forms. The wide variety of projects and research goals in the oceanographic community also results in samples of varying sizes with different precision requirements. Each of the main sample types we analyze requires a unique chemical preparation to convert carbon to $\mathrm{CO}_{2}$ and the potential for introducing carbon during processing differs for each method. Over the past five years, we have developed and refined methods for the chemical preparation of samples and for isotopic analysis of graphite in the accelerator mass spectrometer (AMS). We describe here our methods for preparing samples and we discuss the procedural blanks we measure on the spectrometer. We define a procedural blank as the amount of carbon introduced during the conversion of a sample to graphite.

\section{Methods}

\section{Conversion to $\mathrm{CO}_{2}$}

Inorganic carbon samples are hydrolyzed to $\mathrm{CO}_{2}$ in a side-arm reaction vessel. When there is enough sample material $(>5 \mathrm{mg} \mathrm{CaCO}$ ), samples are weighed into a beaker, treated for $30 \mathrm{sec}$ with a $10 \% \mathrm{HCl}$ solution, rinsed three times with carbon-free water, dried and reweighed. The acid pretreatment removes from $12-36 \%$ of the sample. For small samples $\left(<5 \mathrm{mg} \mathrm{CaCO}_{3}\right)$, we omit the acid pretreatment step. The samples are transferred to reaction vessels, $100 \% \mathrm{H}_{3} \mathrm{PO}_{4}$ is added to the sidearm and the vessel is evacuated and sealed. After sealing, acid is tipped onto the sample and the reaction vessel is maintained at $60^{\circ} \mathrm{C}$ overnight. We use IAEA C-1 (Carrara marble) as our hydrolysis procedural blank, referred to as the hydrolysis blank. We prepare C-1 identically to our samples 
and prepare one $\mathrm{C}-1$ with each batch of 10 samples. Until recently, we used C-1 to determine the AMS machine background and, thus, have our largest data set for this substrate.

Organic samples are oxidized in a closed-tube combustion. Samples ready for combustion are weighed into 9-mm OD $\times 20-\mathrm{cm}$ long Vycor round-bottomed combustion tubes. We have used two procedures to prepare our combustion tubes. For both procedures, the Vycor combustion tubes are precombusted at $850^{\circ} \mathrm{C}$ for $5 \mathrm{~h}$. In the first procedure (Method I), batches of the oxidant ( $\mathrm{CuO}$ in wire form) were prepared by baking at $850^{\circ} \mathrm{C}$ for $3 \mathrm{~h}$ and stored in a desiccator until added to an individual combustion tube. Batches were used over a 2-4 month period before the preparation of a new batch. In the second procedure (Method II), we prepare the combustion tubes by adding $2 \mathrm{~g}$ $\mathrm{CuO}$ (wire form) and placing the tubes in a muffle furnace at $850^{\circ} \mathrm{C}$ for $5 \mathrm{~h}$. The tube is filled with a sample and $100 \mathrm{mg} \mathrm{Ag}$ powder as soon as possible after the tube has cooled, then it is evacuated and flame-sealed. If it is not possible to use the prepared tubes immediately, they are stored evacuated on a vacuum line. When possible, we use enough sample to produce $c a .80 \mu \mathrm{mol}$ carbon. The sample is placed in a muffle furnace at $850^{\circ} \mathrm{C}$ for $5 \mathrm{~h}$. We have used two carbon powders (NBS-21 graphite and Johnson-Matthey $99.9999 \%$ pure carbon powder) as our combustion procedural blanks, referred to as organic combustion blanks. The carbon powder is first weighed into a 6-mm $\mathrm{OD} \times 5$-cm-long precleaned Vycor tube, transferred to a combustion tube and then treated identically to our samples.

Seawater samples are stripped in the bottle in which they are collected; the method is discussed in detail in McNichol et al. (1994). In a nitrogen-filled glove bag, a stripping probe is attached to a sample bottle. The bottle is then attached to our seawater stripping vacuum line, the sample is acidified with $85 \% \mathrm{H}_{3} \mathrm{PO}_{4}$ acid, the line is filled to $0.8 \mathrm{~atm}$ with nitrogen and $\mathrm{CO}_{2}$ is stripped from each sample using nitrogen as a carrier gas. Development of an appropriate procedural blank, which we refer to as a line blank, has been difficult. We discuss here the results of two different methods. In the first method, a stripping probe is attached to an empty sample bottle. The bottle is placed on the vacuum line and evacuated. An aliquot of ${ }^{14} \mathrm{C}$-dead $\mathrm{CO}_{2}$ similar to the quantity we recover from seawater samples is transferred to the evacuated bottle. The bottle and stripping line are brought to 0.8 atm with nitrogen while the $\mathrm{CO}_{2}$ is still frozen down. The treatment of the line blank is then identical to that of the samples. In the second method, a measured aliquot of ${ }^{14} \mathrm{C}$-dead $\mathrm{CO}_{2}$ in a valved vessel is attached to a stripping probe. The probe is then attached to an empty bottle in the same glove bag in which the samples are prepared and the bottle is attached to the vacuum line. At this point, the gas is released into the bottle and the line blank is then treated identically to our samples.

\section{Conversion to Graphite}

All $\mathrm{CO}_{2}$ samples are reduced to graphite over Fe powder using $\mathrm{H}_{2}$ as the reducing agent (Vogel et al. 1987; McNichol et al. 1992). Our line blanks ranged in size from $600-1200 \mu \mathrm{mol} \mathrm{CO} 2$. For our hydrolysis and organic combustion blanks, we produced $\mathrm{CO}_{2}$ from the parent material in quantities ranging from 20 to $800 \mu \mathrm{mol}$. For very large samples, we either split the gas into smaller portions from which we made small individual batches of graphite or we made one large batch of graphite, which was later pressed into many targets. We report here an individual result for each separate target run on the AMS as well as the average of all results for one individual hydrolysis or combustion reaction. We assume that most of the procedural blank is added during reaction to $\mathrm{CO}_{2}$ and that graphite reduction makes a minor contribution to the overall blank (Vogel et al. 1987). 


\begin{abstract}
AMS Analysis
Approximately $2 \mathrm{mg}$ of the graphite/catalyst mixture for each sample is poured into a drilled aluminum cartridge, where it is pressed against a ground, flat surface with a pressure of $4.6 \mathrm{kbar}$ by an automated press. The diameter of each target is $1.5 \mathrm{~mm}$. We have recently started using JohnsonMatthey $99.9999 \%$ pure carbon powder (hereafter referred to as JME powder) to evaluate our machine background. It is used directly from the bottle and pressed with no additional ingredients.

The samples, standards and blanks are loaded into a carousel with 59 load positions. One position is left empty, and a solid aluminum cartridge is loaded into another position. The carousel is placed into the ion source air lock, where it is pumped down, and outgassed for several hours. After a cleaning cycle under the cesium sputter beam, data acquisition commences with all targets being sputtered in turn. Up to nine exposures are made to each target, over a period of two days. The fraction modern $(\mathrm{fm})$ values reported here are averages of data acquired during all target exposures.

During data analysis, time-interpolated values of the machine background are used for subtraction from the standards and some of the samples (Schneider et al. 1994). For this reason, JME powder targets are measured regularly throughout data acquisition. Residual gases in the ion sources can lead to higher machine backgrounds, so data acquisition is not begun until pressure in the ion source is below $3 \times 10^{-6}$ mbar. Periodic cleaning of the ion sources is also necessary.
\end{abstract}

\title{
RESULTS AND DISCUSSION
}

We present data collected on the accelerator from June 1992 to June 1994. During this period, we established routine protocols for operations in the SPL and the AMS. We have not subtracted the machine background from any of the data reported in the following sections except where noted.

\section{Inorganic Carbon}

Data from the analyses of hydrolysis blanks and JME carbon powder are presented in Figure 1. Until we switched to JME carbon powder, we used the hydrolysis blank to determine the AMS machine background. Results are plotted as a function of the serial target press (TP) number and quantity of $\mathrm{CaCO}_{3}$ reacted. The TP number increases sequentially for each target pressed for AMS analysis. There is a general decrease in $\mathrm{fm}$ values over time with the highest values observed below TP number 1350 and the lowest values above TP number 3810. Most of the fluctuations in the hydrolysis blank data are probably due to variations in the ion source pressure, with outgassing and gettering of products sputtered from the various targets. The range of fm values observed for the hydrolysis blank is between $0.00060(59,600 \mathrm{yr})$ and $0.00932(37,600 \mathrm{yr})$. The average of our analyses since TP number 3810 is $0.00208 \pm 0.00045(49,600 \mathrm{yr})$. There appears to be a significant reduction in the hydrolysis blank and a concurrent improvement in the precision above TP number 3810 , which occurred after the repair of a minor leak in the ion source. Before this improvement, we believe that most of the scatter observed in the hydrolysis blank data was related to accelerator operation, i.e., the machine background, because we continued to see improvement in measured blank values although we have used the same hydrolysis procedure for $>5 \mathrm{yr}$ (Gagnon and Jones 1993). There appears to be no relation between the quantity of $\mathrm{CaCO}_{3}$ hydrolyzed and the measured fm (Fig. 1B) in the range of sample sizes analyzed, indicating that we are not adding gross amounts of contamination during hydrolysis. If we were introducing a measurable amount of modern contaminant to our hydrolysis blanks, we would expect fm to increase as the sample size decreased. 

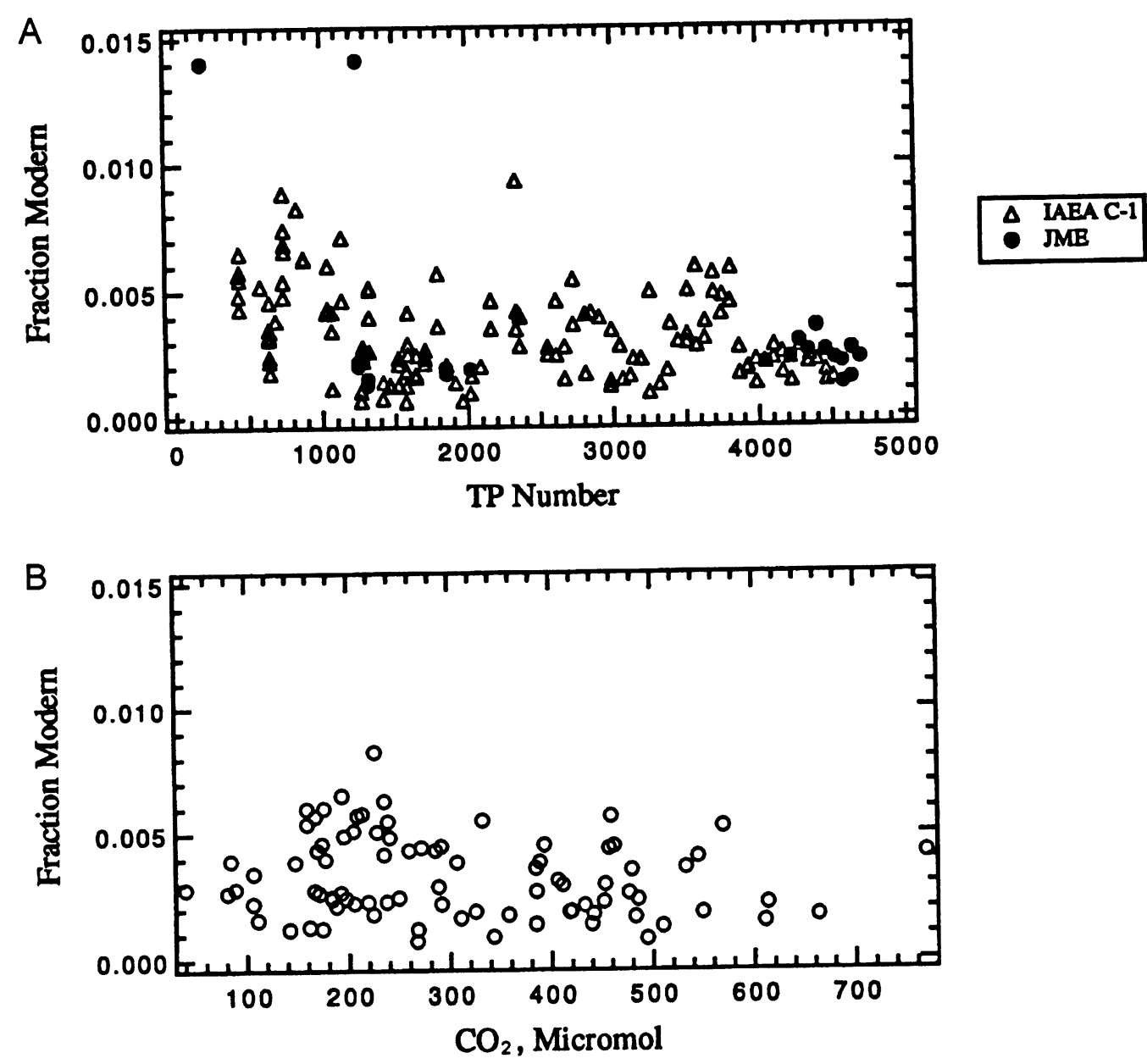

Fig. 1. Fraction modern data for IAEA C-1 (Carrara marble) hydrolysis blanks and JME carbon powder. The JME powder was pressed directly into a cartridge without any sample processing.

Recent analyses of JME carbon powder suggest that it produces a consistently lower machine background than the hydrolysis blank; for the five most recent analyses, the average $\mathrm{fm}$ is $0.002334 \pm$ $0.00061(48,700 \mathrm{yr})$, with a range of 45,300 to $53,500 \mathrm{yr}$. Further analyses of both the hydrolysis blank and JME carbon powder will enable us to estimate the procedural blank introduced during hydrolysis. As we analyze larger quantities of older samples, we will focus more attention on the sources of the machine background and work to reduce them even further.

\section{Organic Carbon}

Figure 2 shows data from the analyses of combusted NBS-21 and JME carbon powder. The fm values reported in Figure 2A were corrected for the introduction of the organic combustion blank using the following mass balance: 
where

$$
f_{s}=\left(f_{m} C_{m}-f_{c} C_{c}\right) / C_{s}
$$

$f_{m}, f_{s}, f_{c}=$ measured, sample, and organic combustion blank fraction modern, respectively, and

$\mathrm{C}_{\mathrm{m}}, \mathrm{C}_{\mathrm{s}}, \mathrm{C}_{\mathrm{c}}=$ amount of measured, sample, and organic combustion blank carbon, respectively.

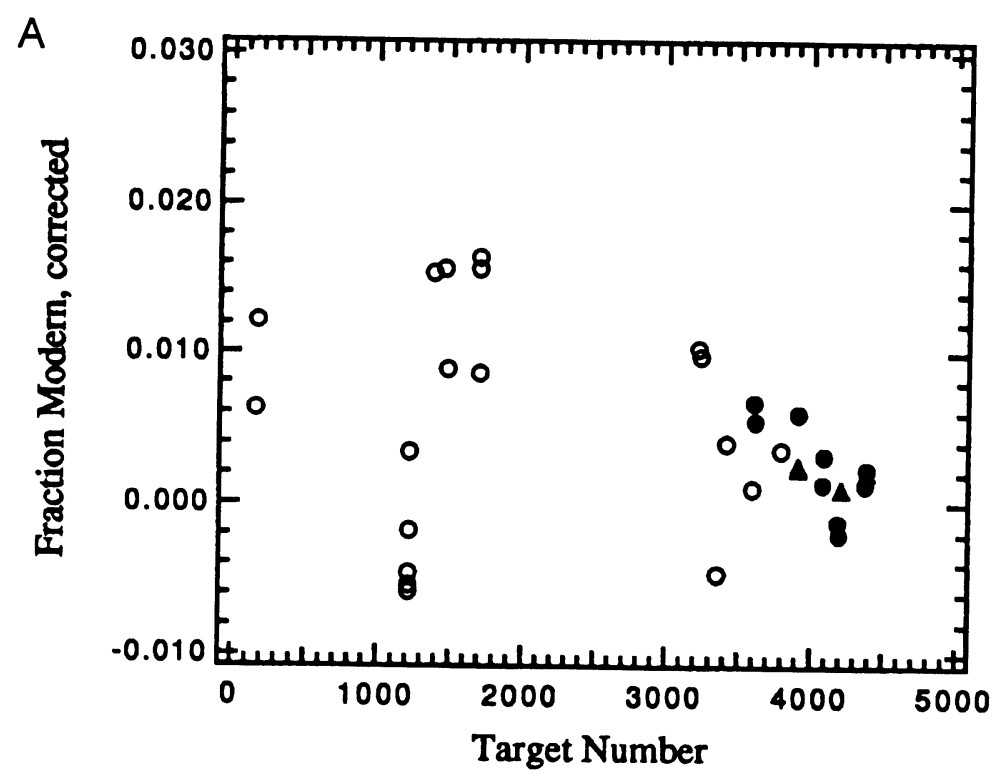

O NBS-21, Method I

- NBS-21, Method D

- JME, Method II

B

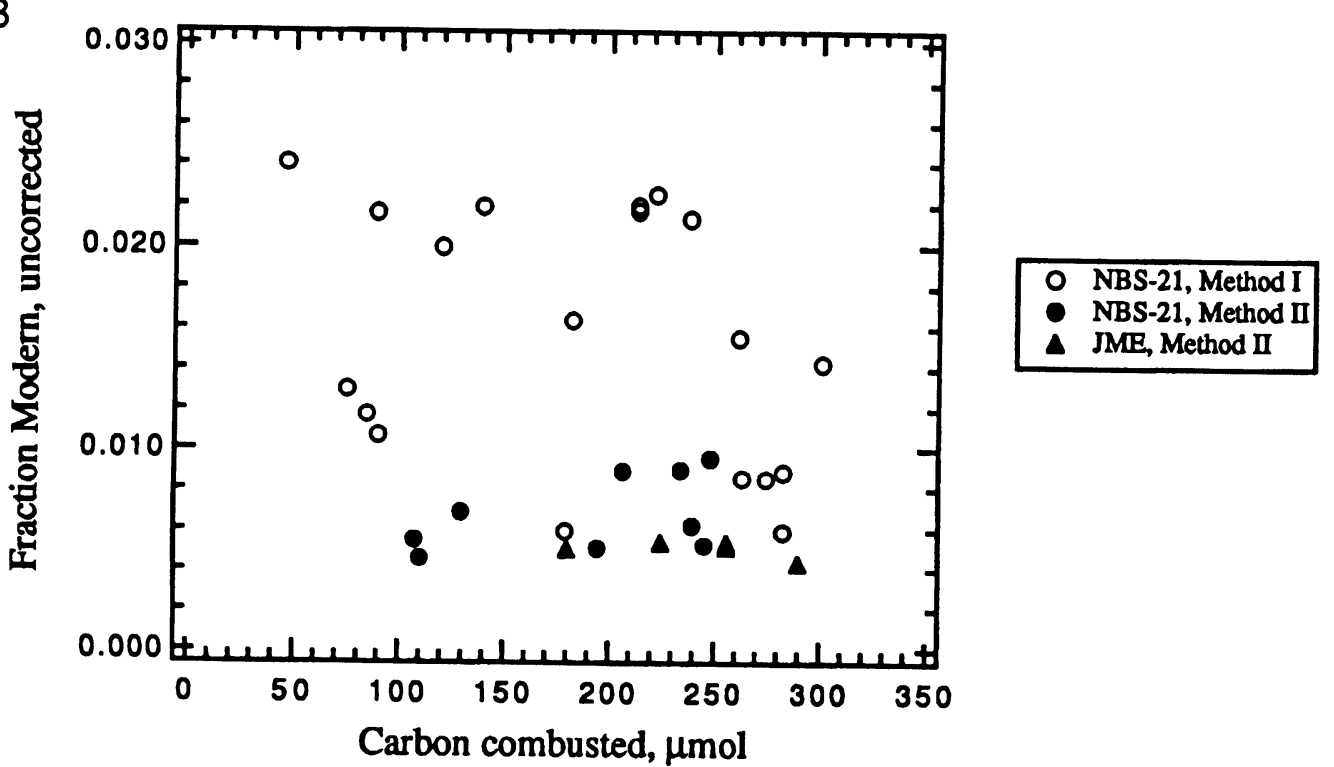

Fig. 2. fm data for oceanic combustion blanks; data are presented for combustions using Method I and Method II. 
We assume that the organic combustion blank is modern (i.e., $\mathrm{fm}=1)$ and that the majority of the carbon is added during combustion of the sample. We quantified the organic combustion blank from each method by combusting empty combustion tubes and measuring the $\mathrm{CO}_{2}$ generated (Table 1) in a small, calibrated volume using a sensitive, accurate pressure transducer. The values reported in Table 1 are significantly greater than those reported elsewhere (Vogel et al. 1987; Donahue et al. 1990). We believe this may be related to the relatively large size of our combustion tubes. Vogel $e t$ al. (1987) suggested that much of the carbon contamination contributed during sample combustion arises from gas adsorbed to Vycor glass. Increasing the Vycor surface area by using a larger tube should increase the contribution from this source. We use larger tubes than most researchers because many of the organic samples we process contain only $0.5-2 \%$ organic carbon by weight, and we must place up to $1000 \mathrm{mg}$ material in a tube. It is apparent from Table 1 and Figure 2 that our organic preparation has been improved by changing the technique for preparing combustion tubes. Considering the fm data from the old method, it appears that we have not constrained the contribution from the organic combustion blank well; we have underestimated it in some cases and overestimated it in others, particularly in the earlier samples. Using the old method, we calculate an average $\mathrm{fm}$ of $0.00568 \pm 0.00769(41,500 \mathrm{yr})$. With the new method, we calculate averages of $0.00254 \pm 0.00307$ $(48,000 \mathrm{yr})$ for NBS-21 and $0.00198 \pm 0.00060(50,000 \mathrm{yr})$ for JME carbon. Considering the uncertainty, these values are similar to those observed for the machine background, confirming our assumption that the organic combustion blank is more important than the graphite preparation blank or the machine background. In Figure 2B, we have plotted the uncorrected values of fm against the amount of carbon combusted. Figure $2 \mathrm{~B}$ further emphasizes how changing our analysis procedure has reduced the size of the blank as well as demonstrates that, for the range of sample sizes we have analyzed, that there is no relation between the quantity of the organic matter combusted and the measured $\mathrm{fm}$. As with the hydrolysis blanks, this is partly because we have not analyzed many small samples.

TABLE 1. Measurement of Combustion Process Blank: Values

\begin{tabular}{|c|c|c|}
\hline (Batch & $\begin{array}{l}\text { Method I } \\
\text { eparation of } \mathrm{CuO} \text { ) }\end{array}$ & $\begin{array}{c}\text { Method II } \\
\text { (Individual preparation of } \mathrm{CuO} \text { ) }\end{array}$ \\
\hline & $\begin{array}{l}1.30 \\
0.83 \\
1.04 \\
1.57 \\
1.31 \\
1.57 \\
1.04\end{array}$ & $\begin{array}{l}0.84 \\
0.85 \\
0.84 \\
0.51 \\
0.88 \\
0.69 \\
0.54 \\
0.72 \\
0.59\end{array}$ \\
\hline $\begin{array}{l}\text { Mean } \\
\text { sd }\end{array}$ & $\begin{array}{l}1.38 \\
0.29\end{array}$ & $\begin{array}{l}0.72 \\
0.14 \\
\end{array}$ \\
\hline
\end{tabular}

\section{Dissolved Inorganic Carbon}

Figure 3A shows data from the water stripping line blanks. We plotted these values with respect to TP number and did not analyze the relationship to quantity of $\mathrm{CO}_{2}$ extracted because we analyze such a large sample ( $c a .1 \mathrm{mmol}$ of $\mathrm{CO}_{2}$ ) that sample size relations are not meaningful. There are three distinct regions of the graph. The values below TP number 2100 are relatively scattered and reflect results from several different methods that we were investigating to prepare our line blanks. 

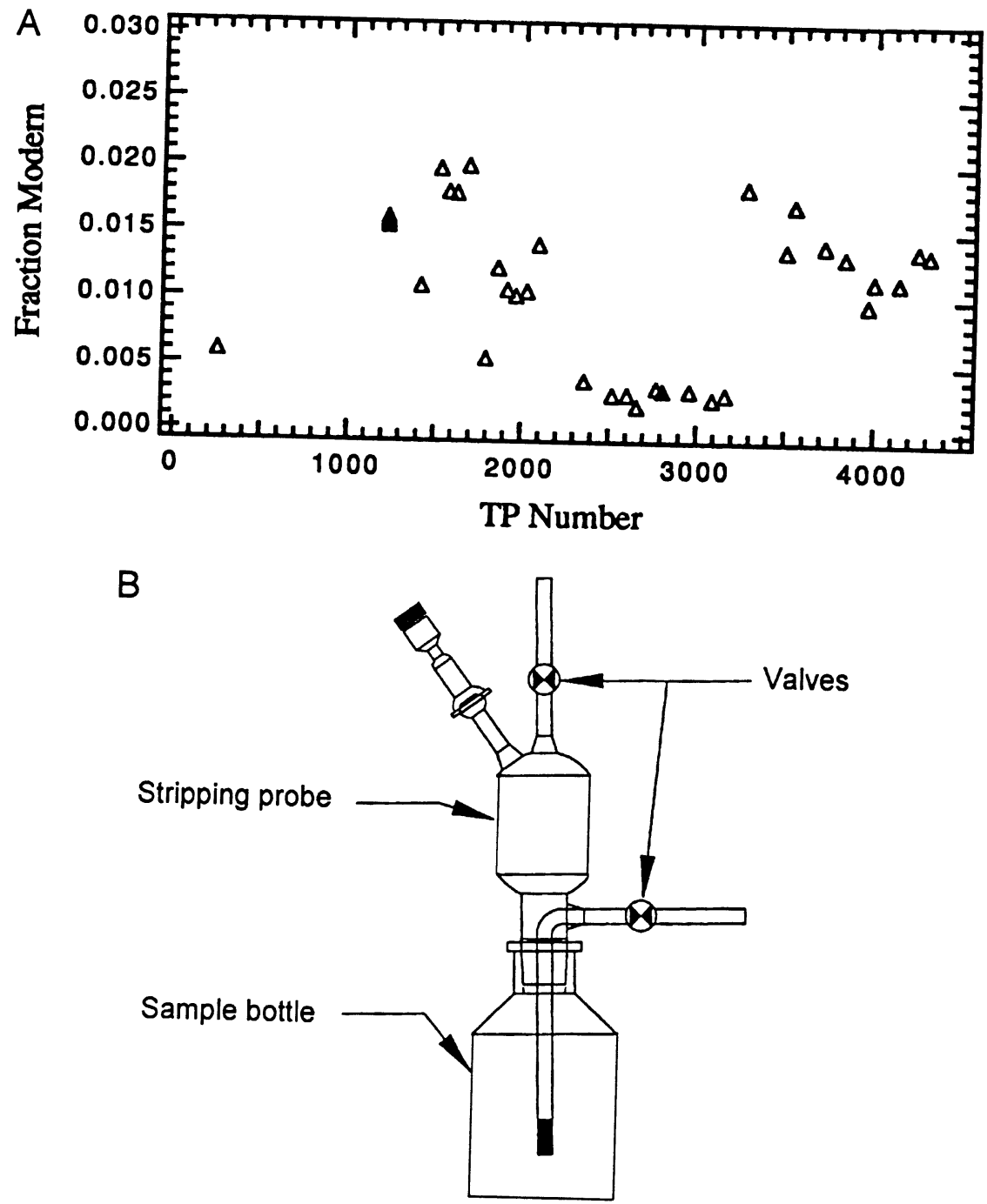
Fig. 3. A. fm data for water stripping line blanks; B. schematic of water-sample bottle with
stripping probe attached

During this time, we used the line blank primarily to verify that no contaminated samples were being stripped on the water line. The values between TP number 2100 and 3200 correspond to line blanks prepared using the first method described. The average fraction modern is $0.00286 \pm 0.00053$, which is similar to $0.00126 \pm 0.00165$, the value we have measured by analyzing the tank $\mathrm{CO}_{2}$ directly. The values shown for TP numbers $>3200$ have an average $\mathrm{fm}$ of $0.01360 \pm 0.00259$, signif$\mathrm{CO}_{2}$ added to the that obtained with the first method. The increase corresponds to the amount of As shown in Figure 3B when attaching the stripping probe to the sample bottle in the glove bag. preparing our line blathe stripping probe and sample bottle have significant volumes that, in the glove bag. Using a maspere, but have apparently entrained some $\mathrm{CO}_{2}$ from the laboratory air in 
ern, we calculate that we add $c a .10 \mu \mathrm{mol} \mathrm{CO}$ to the line blank. Preparation of the line blank isolates almost $800 \mathrm{ml}$ of the glove bag atmosphere in the empty sample bottle and stripping probe volume. With actual seawater samples, we isolate only $300 \mathrm{ml}$ in the stripping probe volume. Thus, only 4 $\mu \mathrm{mol}$ of $\mathrm{CO}_{2}$ are added to each sample. The addition of this carbon to even the most depleted seawater sample $(\mathrm{fm}=0.7)$ will alter the measured $\mathrm{fm}$ by $<2 \%$, within the error we measure. We believe that most of the line blank is added to the water samples during the glove bag operations. One other step in the water stripping process whose blank contribution is being quantified is the addition of acid through a septum in the stripping probe.

\section{SUMMARY}

A summary of data from the process blanks is shown in Figure 4 and Table 2.

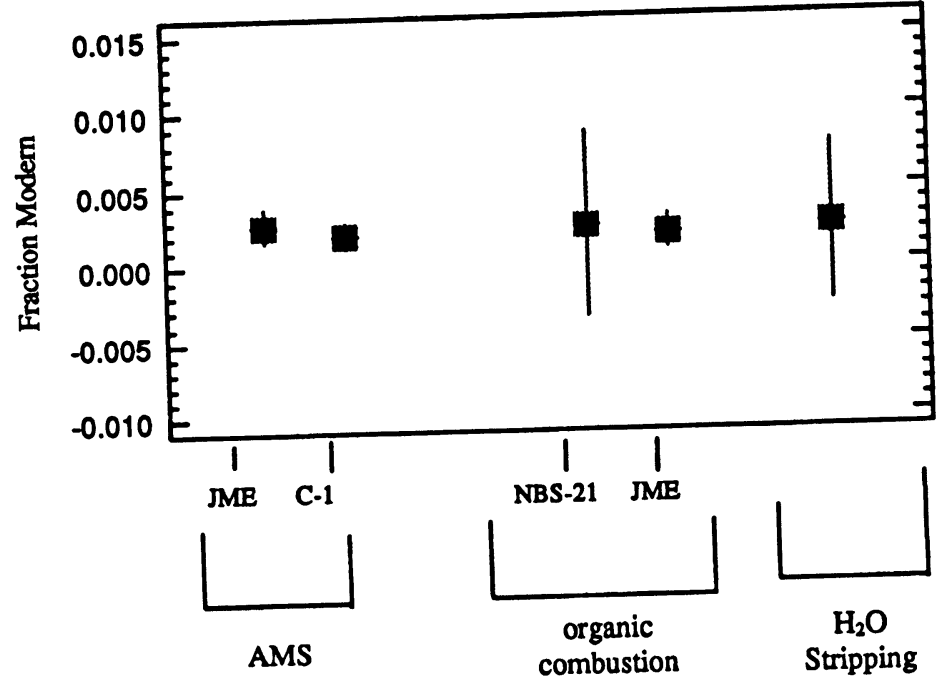

Fig. 4. Summary of corrected process blanks; error bars represent $2 \sigma$

TABLE 2. Summary of current measured process blanks after correction (see text). The amount of carbon added during processing is listed for each process. For $\mathrm{fm}$ values $1 \sigma$ error is reported, and the number of analyses included in the average is reported in parentheses.

\begin{tabular}{|c|c|c|}
\hline Procedure & $\mu \mathrm{mol} \mathrm{C}$ & $\mathrm{fm}$ \\
\hline AMS machine & & \\
\hline IAEA C-1 & -- & $0.002334 \pm 0.00061(12)$ \\
\hline JME carbon powder & -- & $0.002660 \pm 0.00056$ \\
\hline Hydrolysis & -- & Not determined \\
\hline $\begin{array}{l}\text { Organic combustion } \\
\text { NBS- } 21\end{array}$ & & $0.00254 \pm 0.00307$ \\
\hline $\begin{array}{l}\text { NBS-21 } \\
\text { JME carbon powder }\end{array}$ & $\begin{array}{l}0.7 \\
0.7\end{array}$ & $0.00198 \pm 0.00060$ \\
\hline DIC & $10^{*}$ & $0.00257 \pm 0.00260(10)$ \\
\hline
\end{tabular}

*This value refers only to carbon added to the line blank; see text for amount added to water samples. 
The fm values presented here are those calculated by subtracting the contribution from any procedural blank introduced during sample preparation. With this correction, it is not possible to distinguish any process blank from the AMS machine background, verifying that we have accurately quantified the amount of carbon added during sample handling.

\section{Conclusion}

We analyzed procedural blanks for the three most common sample preparation methods we use at NOSAMS. We routinely measure $\mathrm{fm}$ values between 0.0020 and 0.0025 for ${ }^{14} \mathrm{C}$-free hydrolysis samples. After correction for the organic combustion blank, we measure similar values for ${ }^{14} \mathrm{C}$ analyses.

\section{ACKNowledgments}

We thank Greg Cohen for hard work in the engineering and AMS labs and John Vogel for critical review of the manuscript. This work was supported by grants OCE-8802509 and OCE-9301015 from the National Science Foundation. This is WHOI contribution number 9040.

\section{REFERENCES}

Donahue, D. J., Linick, T. W. and Jull, A. J. T. 1990 Isotope-ratio and background corrections for accelerator mass spectrometry radiocarbon measurements. $R a$ diocarbon 32(2): 135-142.

Gagnon, A. R. and Jones, G. A. 1993 AMS-graphite target production methods at the Woods Hole Oceanographic Institution during 1986-1991. Radiocarbon 35(2): 301-310.

McNichol, A. P., Gagnon, A. R. Jones, G. A. and Osborne, E. A. 1992 Illumination of a black box: Analysis of gas composition during graphite target preparation. In Long, A. and Kra, R.S., eds., Proceedings of the 14 th International ${ }^{14} \mathrm{C}$ Conference. Radiocarbon 34(3): 321-329.
McNichol, A. P., Jones, G. A., Hutton, D. L. and Gagnon, A. R. 1994 The rapid preparation of seawater $\mathrm{\Sigma CO}_{2}$ for radiocarbon analysis at the National Ocean Sciences AMS Facility. Radiocarbon 36(2): 237-246.

Schneider, R. J., Jones, G. A., McNichol, A. P., von Reden, K. F., Elder, K. L., Huang, K. and Kessel, E. D. 1994 Methods for data screening, flagging and error analysis at the National Ocean Sciences AMS Facility. Nuclear Instruments and Methods in Physics Research B92: 172-175.

Vogel, J. S., Nelson, D. E. and Southon, J. R. $1987{ }^{14} \mathrm{C}$ background levels in an accelerator mass spectrometry system. Radiocarbon 29(3): 323-333. 